Journal de la Société des américanistes

Journal de la Société

des américanistes

92-1 et $2 \mid 2006$

tome $92, n^{\circ} 1$ et 2

\title{
Le nahualli-tlahuipuchtli dans le monde nahuatl
}

\section{Roberto Martínez González}

\section{OpenEdition}

Journals

Édition électronique

URL : https://journals.openedition.org/jsa/3127

DOI : 10.4000/jsa.3127

ISSN : $1957-7842$

Éditeur

Société des américanistes

Édition imprimée

Date de publication : 1 juin 2006

Pagination : 111-136

ISSN : 0037-9174

Référence électronique

Roberto Martínez González, «Le nahualli-tlahuipuchtli dans le monde nahuatl », Journal de la Société des américanistes [En ligne], 92-1 et 2 | 2006, mis en ligne le 15 janvier 2012, consulté le 03 septembre 2022. URL : http://journals.openedition.org/jsa/3127 ; DOI : https://doi.org/10.4000/jsa.3127 
LE NAHUALLI-TLAHUIPUCHTLI DANS LE MONDE NAHUATL

\author{
Roberto MARTÍNEZ GONZÁLEZ *
}

Parmi les « quarante magiciens du monde nahuatl » identifiés par López Austin (1967), l'un des personnages les moins connus est sans doute le tlahuipuchtli, terme qui aujourd'hui se trouve associé à des sorcières suceuses de sang. L'intention principale de cet article sera donc de définir la signification et la fonction sociale de la croyance à ce type d'être surnaturel. Étant donné que ce personnage est très vaguement décrit dans les sources anciennes, il faudra rechercher ce qu'en disent les données ethnographiques contemporaines pour le définir et en expliquer le sens. Nous passerons d'abord en revue les informations anciennes, pour ensuite élaborer le stéréotype du tlahuipuchtli contemporain et montrer de quelle façon l'image de la sorcière espagnole intervient dans la construction de l'imaginaire du tlahuipuchtli. Finalement, on tentera d'expliquer son symbolisme et de déterminer si la croyance en des sorciers suceurs de sang existait à l'époque préhispanique. [Mots clés: nahualli, sorcellerie, vampirisme, croyances nahua.]

El nahualli-tlahuipuchtli en el mundo náhuatl. Entre las « cuarenta clases de magos », identificadas por López Austin (1967), uno de los personajes peor conocidos es, sin duda, el tlahuipuchtli, un término que, en la actualidad, es usado para designar a una suerte de bruja chupa-sangre. Considerando tal situación, la intención principal de este artículo será definir el significado y la función social de la creencia en este tipo de entidad sobrenatural. Mas, siendo que, en las fuentes antiguas, el tlahuipuchtli es descrito de manera muy vaga, para su definición, será necesario comparar las informaciones antiguas con los datos procedentes de documentos etnográficos contemporáneos. Así pues, comenzaremos por hacer una revisión de los datos proporcionados por los textos del siglo Xvı para, posteriormente, definir el estereotipo contemporáneo del tlahuipuchtli y describir el modo en que la imagen de la bruja española intervino en la construcción del imaginario del tlahuipuchtli. Por último, procuraremos explicar el simbolismo de este personaje y determinar si la creencia en brujos-chupa sangre existió en la época prehispánica. [Palabras claves : nahualli, brujería, vampirismo, creencias nahuas.]

The nahualli-tlahuipuchtli in the nahuatl world. One of the least known characters in Lopez Austin's (1967) article "Forty magician classes in the nahuatl world ", is the

* Instituto de Investigaciones Antropológicas, UNAM. Avenida del Imán 660, Ed 30-501. Col. Pedregal del Maurel. CP 04720, Coyoacan, México DF [nahualogia@yahoo.com.mx].

Journal de la Société des Américanistes, 2006, 92-1 et 2, pp. 111-136. (Société des Américanistes. 
tlahuipuchtli, a name given actually to some kind of bloodsucking witches. The goal in this work is to define the meaning and function of the belief in this kind of supernatural beings. If we consider that the sixteenth century sources do not present an exhaustive description of this supernatural character, then we must compare the ancient information with recent ethnographic data in order to explain and define its meaning. In the first part of the article, we review the ancient data; secondly we attempt to define the contemporary stereotype of the tlahuipuchtli and then describe how the Spaniard witchcraft concepts participated in the construction of modern tlahuipuchtli image. In the last section of this work, we define its symbolism in order to evaluate if the belief in bloodsucking witches existed in pre-hispanic times. [Key words : nahualli, witchcraft, vampirism, nahuatl beliefs.]

\section{Le TLAHUIPUCHTLI DANS LES SOURCES ANCIENNES}

L'origine et la signification du terme tlahuipuchtli restent incertaines. López Austin (1967, p. 93) propose la signification de « brasero lumineux ». Pourtant si l'on considère que tlahuipuchtli est composé du verbe tlahuia, " allumer une torche " ${ }^{1}$, et de pochtli, "fumée, brume légère " (Wimmer 2003), ce terme pourrait vouloir dire en réalité « fumée ou brume lumineuse ». Une autre possibilité, qui m'a été suggérée par Sybille de Pury (2003, communication personnelle), consiste à considérer le mot pochtli non pas dans son sens de "fumée, brume... ", mais dans celui de "jeune personne » : on le retrouve notamment dans des expressions comme telpochtli, "jeune homme» et ichpochtli, "jeune fille »; tlahuipochtli pourrait alors signifier « jeune personne lumineuse ».

Les informateurs de Sahagún (Codex de Florence, Iv, p. 43) rapportent que le nahualli ${ }^{2}$ " était un tlahuipuchtli ; quelqu'un qui s'enlève la [ou les] jambe[s], vraiment c'était ça son travail » ${ }^{3}$. Dans d'autres sources, le tlahuipuchtli est mentionné comme étant un type de nahualli capable de se changer en feu (Torquemada 1943-1944, II, p. 83). On dit aussi que les tlahuipuchtin « marchent durant la nuit, jettent du feu par la bouche et font peur à qui ils veulent [faire du] mal, de sorte qu'ils [les effrayés] deviennent fous ou meurent. Ils se promènent dans les montagnes pendant la nuit et portent du feu sous la forme d'une torche en flammes... » (Bautista 1965, p. 152). Les tlahuipuchtin « disaient apparaître comme des feux dans la montagne et que, tout de suite, ils voient ce feu à un autre endroit... » (Mendieta 1945, p. 119). Malinalxochitl, la sœur de Huitzilopochtli, est devenue - ou s'est transformée en-tlahuipuchtli, "ihuan tlahuipuchtli mocuepa... ", mais aucune explication n'est donnée sur ce à quoi elle ressemblait ou sur ce qu'elle faisait ainsi transformée (Tezozómoc 1991, p. 28). Dans un autre passage du Codex de Florence (IV, p. 101), il est dit à propos de ceux qui naissaient sous le signe 1-Pluie : « si c'était une femme, elle serait mometzcopinqui, celle qui s'enlève les jambes... ". Ce qui pourrait impliquer un certain rapport entre la féminité et le fait de s'enlever les jambes. Cependant, rien ne permet de dire 
précisément ce que l'on faisait en acquérant cette forme. On peut juste affirmer que le tlahuipuchtli préhispanique était un type de nahualli (ou de femme-nahualli) qui était censé(e) s'enlever les jambes et apparaître sous forme d'un feu - ou portant un feu - dans les montagnes pour faire peur aux gens.

\section{LE NAHUALLI-TLAHUIPUCHTLI À L'ÉPOQUE CONTEMPORAINE}

Les Nahua de la Huasteca de Veracruz classent le nahualli parmi les différents types de sorciers qui sont désignés par le terme tetlachihuijquetl. Sandstrom (1991, pp. 234, 251), à qui nous devons cette information, explique que « certains sorciers peuvent se changer à volonté en animaux (particulièrement en oiseaux) pour faire du mal [...]. Ils ont aussi le pouvoir de se transformer en un être, qui ressemble à un oiseau, appelé nahuali [...]. Le nahuali suce le sang des gens quand ils dorment... ». L'existence des nanahualtin, suceurs de sang et « mangeurs de cœurs "), est également attestée à Xalitla, Guerrero (Goloubinoff 1994, pp. 579581). À Milpa Alta et dans les montagnes de Puebla, il existe aussi un type de nahualli, appelé tlacihque ou tlahuepoche, voué à sucer le sang des enfants (Zantwijk 1960, p. 58 ; Chamoux 1989, p. 307). Enfin, chez les Nahua de la région de Cuetzalan, plusieurs récits évoquent des « apparitions d'animaux qui sucent le sang... ». Ces animaux sont appelés nahuales (Vélez Cervantes 1996, p. 34).

Dans d'autres régions nahua, le tlahuipuchtl - aussi appelé tlahuelpuchi ${ }^{4}$, chixtle $^{5}$, tlahuilpuche, mazacat, brujas, tlacique et tzitzime - est un personnage indépendant du nahualli. C'est le cas par exemple dans la vallée de Tlaxcala (Nutini et Forbes 1987, p. 343), à Chalco-Amecameca (Fabregas Puig 1969, p. 107) ${ }^{6}$ et dans le sud du Veracruz (Münch 1983, p. 375), tandis qu'ailleurs - à Milpa Alta (Zantwijk 1960, p. 58 ; Madsen 1960, p. 203), dans la région de Cuetzalan (Signorini et Lupo 1989, p. 86) et dans la Sierra Norte de Puebla (Montoya Briones 1964, p. 153) - les deux personnages semblent se confondre ${ }^{7}$. De plus, il s'avère que la croyance à tlahuipuchtli n'est pas exclusive des Nahua, ou des peuples indigènes ${ }^{8}$, mais est répandue un peu partout au Mexique. Mendoza (1952, pp. 285-289) présente des informations provenant de Mexico ${ }^{9}$, Zacatecas, Monterrey ${ }^{10}$, les Etats de Guanajuato, Hidalgo, Mexico, Puebla, Tlaxcala, Michoacan, Guerrero, Oaxaca, Yucatan, Campeche et Veracruz.

La conception nahua du tlahuipuchtli semble être synthétisée dans un récit répandu dans toutes les populations de langue nahuatl et, peut-être, ailleurs.

1. Un homme épousa une femme qui «ne voulait pas dormir avec lui, ne voulait rien manger et sortait pendant la nuit » (Campos 1982, p. 183). Un homme épousa une femme qui lui donnait toujours du sang à manger. « Un jour il pensa : "d'où ma femme obtient-elle le sang ? Nulle part on ne tue des bœufs et je ne lui donne pas d'argent pour qu'elle achète du sang au marché" " (Barrios 1949-1957, p. 59 ; Fabregas Puig 1969, p. 108 ; Pury 1982, p. 80). Une belle femme, qui était en très bonne santé quand elle 
était célibataire, ne pouvait plus sortir la nuit, après son mariage, et « était devenue maigre, pâle, paresseuse... » (Madsen 1960, p. 202).

2. L'homme se rend compte en l'espionnant durant la nuit que la femme est une tlahuipuchtli et découvre la procédure qu'elle suit pour se transformer en animal et obtenir le sang : la nuit tombée, quand le conjoint est en train de dormir, la tlahuipuchtli commence à sauter au-dessus du feu - soit en croix ou en ligne sept fois ou un multiple de sept -, puis elle s'enlève les jambes ${ }^{11}$, les dépose à côté du foyer, pour qu'elles restent bien au chaud, sous le lit ou dans un coin de la maison, se change en animal et part en volant. Puis, la tlahuipuchtli rentre chez elle et vomit le sang dans une marmite (Vélez Cervantes 1996, p. 36 ; Madsen 1960, p. 203 ; Gamio 1922, p. 306 ; Fabregas Puig 1969, p. 108 ; Montoya Briones 1964, p. 173 ; Mendoza 1952, p. 287 ; Barrios $1949-1957$, p. 59 ; Nutini et Roberts 1993, pp. 58-59).

3. La nuit suivante, l'homme récupère les jambes de la femme-tlahuipuchtli et les détruit ou les remet aux autorités. Ne pouvant plus marcher, la tlahuipuchtli est incapable de se défendre ou de s'échapper. Finalement, son capteur la tue, la laisse mourir ou la remet aux autorités locales qui finissent par la tuer ${ }^{12}$.

\section{LE TLAHUIPUCHTLI PROTO-TYPIQUE ET SA TRANSFORMATION}

Selon Fabregas Puig (1969, p. 107), Martínez del Río (1962, pp. 80-81), Barrios (1949-1957, p. 59) et Zavala (2001, p. 31), dans les régions de ChalcoAmecameca, Morelos et San Luis Potosi, la transformation des brujas, lorsqu'elles vont sucer du sang, se produit par l'ajout de certains éléments sur le corps : elles « se mettent des pattes de dindon et des ailes en petate [natte] » et, parfois, une queue de balai. Mendoza (1952, p. 287) évoque le remplacement des jambes par des gourdins ou des pattes de dindon, et celui des yeux par ceux d'un chat ou d'une chouette. Dans ces cas, les tlahuipuchtin se servent d'instruments pour acquérir une forme semblable à celle d'un dindon, tandis que les yeux de chat ou de chouette les aident à améliorer leur vue dans la nuit.

Malheureusement, on ne connaît vraiment pas la signification de l'action d'ôter une jambe. La seule autre référence à ce type d'opération apparaît dans les Procesos de indios... (1912, pp. 179-180, 183 ; Olivier 1997, p. 98) où il est dit à propos de Chantico, la déesse du feu domestique, que son image «était faite de telle façon qu'on pouvait retirer la cuisse et la jambe de l'idole et, avec elles, ils [les Indiens] frappaient la terre, et ainsi, disaient-ils, ils vainquaient, conquéraient et assujettissaient leurs ennemis... ». Pourtant, les faits de ce récit présentent des différences fondamentales. En effet, il apparaît que, dans les Procesos de indios..., il est question d'une idole et non d'une entité animée. De plus, le tlahuipuchtli se désarticule lui-même les jambes, tandis que c'est quelqu'un d'autre qui désarticule les jambes de l'efligie de Chantico.

L'absence d'un pied ou d'une jambe est l'un des attributs caractéristiques de Tezcatlipoca et de ses représentants ${ }^{13}$. D'après Olivier (1997, p. 272), le pied manquant de Tezcatlipoca « représentait, en quelque sorte, le stigmate de la 
faute ${ }^{14}$. Ce même auteur s'appuie sur le fait que, dans la Relación de Michoacán (1977, p. 112 ; Corona Núñez 1946, p. 139), est expliquée la mésaventure de la divinité purépecha, Tares Upeme (la divinité du pulque), qui fut précipitée du haut du ciel sur la terre par les autres dieux et c'est « pour cela [que] ce dieu était boiteux ». Tedlock (1982, p. 110) signale que, pour les Quichés de Momostenango, l'expression c'at rakan (" brûler sa jambe ») qualifie les rapports sexuels adultères. Il existe un récit tepehua dans lequel les personnes décédées qui ne se sont jamais mariées à l'Église n'ont pas de jambes et marchent sur les genoux (Williams García 1972, p. 40). Plus explicitement, Galinier (1984, p. 45) ajoute que, pour les Otomi, le pied symbolise le pénis et la perte de la jambe, la castration. En fait, d'après cet auteur, les hommes qui se transforment en animaux en enlevant leur jambe se changent d'abord en femmes : « cette opération leur permet d'effectuer une transexualisation (d'homme à femme) puis de s'envoler sous l'apparence d'un oiseau... ${ }^{15}$. D'ailleurs, les contes mésoaméricains parlent souvent d'hommes émasculés qui deviennent des femmes (Galinier ibid. ; Barrios 1949-1957, pp. 56, 61).

Quant à la forme qu'ils adoptent le plus souvent, les tlahuipuchtin se changent en dindons ${ }^{16}$, animal qui semble aussi être associé à la répression sexuelle et à la sexualité illicite. Les informateurs de Sahagún (1969, p. 85) disent que les petits dindons risquent de mourir au contact de personnes adultères et que la protubérance sur la tête des dindons rend les hommes impuissants (Codex de Florence, XI, pp. 53-54). D'après Olivier (1997, p. 272), c'est à cause de cette fonction répressive que le dindon était associé à Tezcatlipoca ${ }^{17}$.

Actuellement, dans les montagnes de Puebla, le dindon est un animal associé à la femme. C'est pour cela que, parfois, les personnages des récits compilés par Taggart (1984, p. 98) chez les Nahua de la région de Cuetzalan appellent leurs femmes « ma dinde ${ }^{18}$. Pour les Nahua du centre de Veracruz, le dindon, comme objet de transformation des tlahuipuchtin, est tellement associé à la féminité que même si le sorcier est un homme, l'oiseau est toujours une femelle. On raconte en effet que, ayant entendu le battement des ailes d'un oiseau, les habitants de la maison effectuèrent un rituel leur permettant de le paralyser. Ce fut à ce moment-là qu'ils virent tomber l'énorme dinde qui était posée sur leur toit. Se voyant paralysé, le sorcier-dinde, qui était en réalité le compère du propriétaire de la maison, un homme-tlahuipuchtli, dit : «Non, compère, ne me tues pas !» (Robe 1971, p. 148).

Par ailleurs, dans les données anciennes, le dindon est un animal en rapport avec le feu. Selon la Leyenda de los Soles (1945, p. 119) et l'Histoyre du Mechique de Thevet (Jonghe 1905, p. 23), « ceux qui vécurent durant le troisième [âge] au temps du soleil 4-Pluie périrent aussi, il plut du feu sur eux et ils se transformèrent en dindons... > ${ }^{19}$. La partie essentielle du rite de transformation des tlahuipuchtin contemporains - sauter ou passer au-dessus un feu ${ }^{20}$ - reproduit d'ailleurs le même schéma que le mythe de la Leyenda de los Soles : c'est par le feu que 
l'homme se change en dindon. Cet animal est en même temps associé à la pluie et au tonnerre : dans le Codex Borgia (1963, I, p. 150), il est relié à Tlaloc et à la pluie et préside la treizaine 1-Eau ${ }^{21}$. Chez les Nahua de la région de Cuetzalan, il existe « une sorte de jumeau méchant du Baptiste (divinité locale de la pluie), appelé Ahuehuexcho (dindon d'eau)... [qui] provoquerait d'énormes inondations » (Lupo 2001, p. 350). Un personnage semblable se présente aussi chez les Nahua de Veracruz sous le nom de totole et on affirme que c'est « un être surnaturel qui représente le temps de la saison des pluies. Le mouvement de ses ailes produit le vent, et son chant le tonnerre » (Noriega Orozco 1997, p. 561) ${ }^{22}$. Ce qui semble coïncider avec le fait que, suivant Fabregas Puig $(1969$, p. 109) et Nutini et Roberts (1993, p. 60), la saison des pluies est celle qui convient le plus aux tlahuipuchtin lorsqu'ils vont sucer le sang des enfants.

Une autre caractéristique importante est la luminosité des tlahuipuchtin; la source de lumière peut changer d'une région à l'autre, mais le fait que ce personnage soit visible la nuit est un élément constant ${ }^{23}$. Outre la capacité de transformation, les pouvoirs surnaturels qu'on attribue le plus souvent aux tlahuipuchtin sont la capacité de se rendre invisible (Madsen 1960, p. 202) ou d'obscurcir son environnement afin de pouvoir se cacher (Fabregas Puig 1969, p. 108), le pouvoir d'instiller le sommeil chez leurs victimes (Madsen ibid. ; Nutini et Roberts 1993, p. 66 ; Fabregas Puig ibid.) ${ }^{24}$, celui d'entendre tout ce qu'on dit sur eux (Nutini et Roberts ibid., p. 56) et celui de se faire tout petits pour pénétrer dans les maisons en passant par la serrure (Fabregas Puig ibid. ; Mendoza 1952, pp. 287-288).

Aujourd'hui, la croyance au tlahuipuchtli est répandue dans tout le Mexique, aussi bien chez les Indiens que chez les métis. Généralement, il s'agit d'un être lumineux essentiellement féminin qui, la nuit, se change de préférence en dindon pour aller sucer le sang des enfants. Même si parfois cette métamorphose est censée se produire à l'aide d'instruments, le plus souvent le tlahuipuchtli doit passer par le feu pour s'enlever les jambes et adopter la forme d'un dindon, d'un oiseau de nuit ou d'un autre animal. L'absence de jambes semble être en relation avec le péché sexuel. Le dindon, forme la plus adoptée par les tlahuipuchtin, peut être associé à l'impuissance, à la féminité, à la pluie, au feu, au tonnerre ; et, selon le même schéma que celui du mythe de la destruction du troisième soleil, c'est le feu qui transforme l'humain en dindon.

\section{MOYENS PRÉVENTIFS}

D’après les informateurs de Sahagún (Codex de Florence, v, p. 157 ; Historia General 1938, v, p. 35), une des méthodes employées pour se protéger des nanahualtin-tlahuipuchtin ${ }^{25}$ consistait à mettre une obsidienne dans un récipient rempli d'eau et à le placer derrière la porte ou dans la cour. 
Dans la nuit, avancent les hiboux-hommes [tlacatecolo], peut-être les nanahualtin, les tlahuipuchtin, et ils s'attaqueront aux foyers des gens [...]. Alors [ceux-ci] mettent une obsidienne dans l'eau derrière la porte, peut-être la mettent-ils dans la cour, pendant la nuit. Ils disent : « ici les hiboux-hommes, les tlahuipuchtin se regardent dans le miroir en allant faire du mal aux gens. Mais il [le sorcier] fuit. Il ne fait pas de mal aux gens quand il voit l'obsidienne qui est dans l'eau ».

Ainsi, le fait que le malfaiteur se regarde dans un miroir d'eau et d'obsidienne est une condition nécessaire pour le faire fuir. Si, comme on l'a dit plus haut, en les regardant, les tlahuipuchtin « font peur à ceux à qui ils veulent nuire... » (Bautista 1965, p. 152), il est possible qu'en voyant leur propre reflet dans un miroir l'effet se retourne contre eux. De semblables méthodes sont encore employées par divers peuples nahua ${ }^{26}$. Pour éviter qu'un tlahuipuchtli n'entre dans la maison, « on met, au milieu de la cour, soit un récipient avec de l'eau, soit un miroir sur la porte, soit des ciseaux en croix à l'entrée des chambres... » (Fabregas Puig 1969, p. 109 ; Mendoza 1952, p. 288 ; Fagetti 1996, pp. 215-216). L'usage de substances aromatiques, d'objets en métal, de chapelets bénits, de minuscules répliques des évangiles, de cordelettes rouges est aussi mentionné (Mendoza ibid. ; Nutini et Roberts 1993, p. 70). Quant aux ciseaux, il est possible que ce soit leur forme en croix qui les font fuir car, comme les vampires, les tlahuipuchtin sont des êtres diaboliques qu'un symbole chrétien peut repousser ; de plus, à Tlaxcala, il est connu que les "tlahuelpuchis ont par nature peur des métaux... » (Nutini et Roberts ibid., p. 68$)^{27}$.

Pour les Nahua de Puebla, du centre de Veracruz, de la vallée de Tlaxcala et de la région de Chalco-Amecameca, le moyen le plus efficace pour tuer les tlahuapoches consiste à « enlever leurs vêtements rapidement devant eux et à les mettre à l'envers. Avec cela ils tombent morts ». D'une façon similaire, d'autres Nahua de Chalco-Amecameca et de la vallée de Puebla soutiennent qu'il faut « retourner un chapeau, le jeter par terre et lui clouer un couteau sur la calotte. [Mais] si la sorcière est sur le toit, il suffit de sortir complètement nu pour qu'elle meure [...] on peut arriver à la capturer en récitant le Magnificat mais à l'envers " ${ }^{28}$. On croit aussi que, pour y parvenir, un homme doit lancer un rebozo (une sorte de large écharpe) au tlahuipuchtli et une femme doit faire pareil avec un chapeau (Montoya Briones 1964, p. 174 ; Fabregas Puig 1969, p. 109 ; Robe 1971, p. 147 ; Nutini et Roberts 1993, p. 71 ; Fagetti 1996, p. 216). Ainsi, les moyens de prévention et de neutralisation des tlahuipuchtin peuvent être répartis en deux groupes : celui des moyens à symbolisme chrétien ou d'origine européenne et celui qui provoque des inversions.

\section{TLAHUIPUCHTLI, DE L'IMAGINAIRE À LA REPRÉSENTATION}

Les ethnologues n'ont pas enregistré beaucoup de cas d'individus particuliers accusés d'être tlahuipuchtin ${ }^{29}$. D'après Nutini et Roberts (1993, p. 55), « l'un des 
signes le plus évident pour dire que quelqu'un est tlahuelpuchi est le rôle dominant et possessif joué par la femme dans une relation de couple » ${ }^{30}$.

Nous avons vu que, parmi les moyens préventifs, l'inversion tue le tlahuipuchtli ou le rend immobile. Si nous revenons au récit initial, remarquons que l'histoire a comme point de départ une situation anormale par rapport aux règles qui régissent les relations de couple : une femme qui ne couche pas avec son mari, un homme qui ne donne pas d'argent à sa femme, une femme qui obtient de la nourriture pour son homme, etc. À Tlaxcala, on soupçonne une femme d'être tlahuipuchtli quand elle est dominante, ce qui pourrait être compris comme une inversion des genres ${ }^{31}$. Retenons également que l'activité du tlahuipuchtli se rapproche de la tromperie et de la clandestinité. Il s'agit d'un acte fait en cachette du conjoint. La clandestinité peut clairement devenir infidélité. Un récit nahuatl, recueilli par Campos (1982, p. 183), mentionne le cas d'une femme-tlahuipuchtli qui se transformait en ânesse pour avoir des rapports sexuels avec un diable métamorphosé en âne. Par ailleurs, le tlacique de Milpa Alta a pour caractéristique de ne pas manger de viande. Ce trait se retrouve également dans un conte de la Sierra Norte de Puebla qui parle d'une fille à la sexualité débridée. Sa mère lui offre toutes sortes de viandes qu'elle refuse : « mon Dieu, et alors, quelle viande veux-tu ? » et la fille répond: " je veux de celle avec laquelle les hommes urinent... » (Taggart 1984, pp. 241-243; Madsen 1960, p. 202). À la fin des récits, la punition permet de restaurer l'ordre perdu : le mari détruit les jambes du tlahuipuchtli, l'homme domine la femme... ${ }^{32}$.

Ainsi, le tlahuipuchtli contemporain symbolise, la plupart du temps, une féminité aberrante; il n'est pas un spécialiste rituel, mais une représentation sociale de l'inversion des genres. Une inversion qui, loin de se percevoir seulement dans les méthodes préventives, est explicitement décrite par les informateurs indigènes.

\section{TLAHUIPUCHTLI ET SORCIÈRE}

Les tlahuipuchtin ne se limitent pas à sucer le sang, ils utilisent aussi leurs pouvoirs surnaturels pour attaquer leurs ennemis, pour se venger d'offenses reçues ou, simplement, par envie. Les tlahuipuchtin égarent les gens grâce à l'hypnose ou en provoquant chez eux des hallucinations quand ils sont dans la forêt. Ils les mettent en danger, près d'un précipice, par exemple. " Il cherche à épouvanter les promeneurs attardés, il saccage les récoltes ou s'introduit chez les gens pour faire du mal aux enfants » (Nutini et Roberts 1993, p. 63 pour la région du Tlaxcala ; voir Mendoza 1952, pp. 286-287 pour l'ensemble du Mexique ; Goloubinoff 1994, pp. 579-581 pour la région du Guerrero).

Le tlahuipuchtli est donc un être clairement maléfique pour la communauté. Il est d'ailleurs associé à tout ce qui est démoniaque et, en conséquence, identifié à 
la sorcière espagnole. Dans un récit du Veracruz, la "sorcière » et son mari assistent à un festin de sang humain en compagnie d'autres sorciers - comme si c'était un sabbat ${ }^{33}$ : «Et on dit que là-bas, celui-ci [le mari], lorsque les autres commencèrent à manger, prit une tortilla [et dit :] "Au nom du Père, du Fils et du Saint-Esprit, Amen”. L'endroit se vida. Tous [les gens] disparurent, tous !» (Robe 1971, p. 101) ${ }^{34}$. Ce dernier détail - la mention du Dieu catholique qui met fin à la réunion - se retrouve dans la description d'un sabbat du $\mathrm{XvII}^{\mathrm{e}}$ siècle à Zugarramurdi, en pays basque espagnol: "vu qu'elle était une très bonne danseuse, elle faisait dans sa danse des sauts aussi hauts que les toits des maisons [...] María Irarte admira et s'exclama : “Jésus ! Mais qu'est-ce que c'est ?" À cet instant tout disparut » (Lison-Tolosana 1996, p. 127) ${ }^{35}$. De fait, un informateur de Robe (1971, p. 101) semble faire une référence explicite à l'origine espagnole de la croyance aux sorcières suceuses de sang en disant: "Et la source de la sorcellerie est en Espagne $»{ }^{36}$.

L'image de la sorcière volante fut importée dès les premières années de l'époque coloniale. On en retrouve dans les procès inquisitoriaux à partir de la fin du Xvl ${ }^{\mathfrak{e}}$ siècle. En 1751, une Indienne tlaxcaltèque, nommée María ou Gregoria Borrego, fut accusée d'être sorcière et de voler dans les airs; elle reconnut être hechicera, " ensorceleuse », et admit avoir rencontré le démon, mais « elle répond qu'elle n'a jamais volé, ni ne sait comment voler, ni n'a jamais été bruja [sorcière]... ». Pourtant " en prison, l'Indienne Eugenia dit qu'elle volait quelquefois » (AGN Inquisición 1751, 939.8, fol. 296v) ${ }^{37}$.

Un autre trait qui semble venir de la sorcellerie espagnole est le type de punition appliquée aux tlahuipuchtin que l'on capture. Dans la région de ChalcoAmecameca, «quand on capturait une sorcière ${ }^{38}$, on l'emmenait faire un tour [dans le village] en compagnie d'une bande de musiciens, pendant qu'on préparait le feu où elle allait être brûlée » (Fabregas Puig 1969, p. 109). On retrouve aussi des histoires de tlahuipuchtin brûlés dans la Sierra Norte de Puebla (Montoya Briones 1964, p. 52), à Hueyapan, Morelos (Barrios 1949-1957, p. 60) et dans le sud du Veracruz (Münch 1994, p. 173). Robe (1971, p. 143), Goloubinoff (1994, pp. 609-610) et Refugio Miranda (2002, communication personnelle) font aussi référence au fait de brûler des nanahualtin non considérés comme suceurs de sang. Cependant, il s'agit d'une situation très différente car ce qu'on brûle n'est pas l'être humain, mais son double-nahualli et, lorsqu'on le brûle, il est déjà mort ${ }^{39}$. Il ne s'agit donc pas d'une façon de tuer le malfaiteur, mais d'un moyen pour empêcher que le nahualli ne revive. Tuer une sorcière en la brûlant est un acte typiquement associé à la sorcellerie européenne ${ }^{40}$. Ainsi, ce n'est pas un hasard si plusieurs peuples indigènes traduisent le mot tlahuipuchtli par bruja (« sorcière »); la sorcellerie espagnole fait partie intégrante de leur croyance.

Cependant, une différence importante entre les tlahuipuchtin et les sorcières espagnoles est que, souvent, la capacité maléfique des premiers est considérée comme innée et non optionnelle. Ainsi, par exemple, dans les montagnes de 
Puebla, le mari demandait à la femme-tlahuipuchtli : "Comment fais-tu pour enlever ton pied ? [La femme répond :] Depuis ma naissance j'ai toujours, toujours pu le faire, j'ai grandi comme ça [...]. Mais celui qui ne peut pas le faire, ne pourra jamais le faire ; même s'il le voulait » (Segre 1990, pp. 312-313) ${ }^{41}$. Dans la vallée de Tlaxcala « les tlahuelpuchis naissent avec leurs pouvoirs maléfiques [...]. Si une personne naît tlahuelpuchi, elle conserve ce statut durant toute sa vie... " (Nutini et Forbes 1987, p. 341). On dit encore que « pour subsister, la sorcière a besoin de sang humain... ». Les tlacique de Milpa Alta sont "destinés dès la naissance à développer des pouvoirs surnaturels maléfiques sans aucun entraînement. Vu qu'un vampire [tlacique] ne peut pas manger de viande, il doit se nourrir de sang humain ". Puisque la nature des tlahuipuchtin ne se manifeste qu'à la puberté, c'est à partir de ce moment qu'ils « peuvent se changer en animaux et se sentent envahis par un besoin irrésistible de sucer le sang des enfants, qu'ils doivent satisfaire au moins une fois par mois, sous peine de mort » (Mendoza 1952, p. 286 ; Madsen 1960, p. 202 ; Nutini et Forbes 1987, pp. 341 342). Il est possible qu'à l'époque précolombienne le type de double-nahualli ait été supposé avoir une certaine influence sur l'assignation du rôle de tlahuipuchtli à un individu. Même si, pour les Mixe contemporains, « les sorciers reçoivent leur pouvoir de Luzbel [...], dans les villages les plus traditionnels, le pouvoir de faire du mal aux autres en leur suçant le sang est acquis à la naissance par la possession d'une certaine co-essence animale » (Lipp 1991, p. 161). Pour les Nahua de la vallée de Puebla, ceux qui ont comme nahualli le dindon seront destinés à sucer le sang des enfants (Fagetti 1996, p. 212). Chez les Zapotèques, les sorcières ont toujours le chien comme double (Parsons 1966, p. 133). De la même façon, chez les Totonaques, si un individu a comme double le « coyolite (piçawa) ou faisan sauvage, il est franchement néfaste, suçant l'âme des enfants endormis » (Ichon 1969, p. 179). Au final, même si certaines de ces caractéristiques semblent être empruntées à la sorcière espagnole, le tlahuipuchtli conserve des traits reconnaissables appartenant à son modèle préhispanique.

\section{ExistaIT-IL UN NAHUaLli SUCEUR DE SANG À L'ÉPOQUE PRÉHISPANIQUE?}

Aucune source ancienne n'affirme que le tlahuipuchtli ou le mometzcopinqui suce le sang des enfants ; en revanche, en plusieurs endroits de la Mésoamérique, il est fait mention de sorciers suceurs de sang. Sahagún (1938, X, p. 33) affirme que « le nahualli proprement dit s'appelle sorcier qui, pendant la nuit, effraie les hommes et suce les enfants ${ }^{42}$. Cette caractéristique n'est nulle part ailleurs mentionnée à propos du nahualli, ni dans le Codex de Florence, ni dans aucune autre source aztèque du xvi ${ }^{\mathrm{e}}$ siècle ${ }^{43}$. Ixtlilxochitl (1975-1977, II, p. 54) dit que Tezozomoc rêva que Nezahualcoyotl se transformait en aigle et en tigre, lui mangeait le cœur et buvait son sang. Cependant, parce qu'il ne s'agit que d'un 
rêve prémonitoire, il ne mentionne pas le terme indigène qui aurait fait référence à une telle action. En fait, le seul cas dans lequel un Indien accuse un autre de consommer du sang apparaît dans l'un des procès inquisitoriaux du Chiapas. D'après le texte (AHDCH 1778, fol. 8-9), Sebastián Gómez déclara qu'Antonio Morales lui avait dit « qu'il allait me boire mon sang et celui de Santos Ruiz [...]. À partir de ce moment, mes proches commencèrent à tomber malades d'un mal inexplicable... ».

Molina (1970) traduit « sorcière qui suce le sang " par teyollocuani « celui qui mange les cœurs des gens ». Urbano (1990, fol. 66), dans son Vocabulario Trilingïe..., traduit en nahuatl « sorcier qui suce le sang » par teyollocuani, techichinani " celui qui suce les gens » ${ }^{44}$ et en otomi par nogatzahnate "celui qui mange l'esprit ou souffle vital " ${ }^{45}$, nogatzamamuayte "celui qui mange l'âme ou cœur » ${ }^{46}$. Dans le Diccionario otomi (1640 in Carrasco 1950, p. 224), on trouve " sorcière qui suce le sang se dit : nodiyēttī ; nogazynaqhy ; nogazamamyi; noyagānmague ». Nodiyēttī - terme formé sur la racine "yethi» (" médecine, remède ») ${ }^{47}$ - est un des noms de «l'enchanteur qu'ils adoraient comme un dieu » et un des termes employés par Urbano (ibid.) pour traduire les vocables nahua "nahualli, tizitl». Nogazynaqhy signifie "celui qui suce le sang ou le sperme », nogazamamyi « celui qui suce le principe vital ou le cœur (mbui) » et noyaganmague « celui qui mange la chair » (Galinier 2004, communication personnelle). Dans le Vocabulario matlatzinca de Basalenque (1642 in Carrasco 1950, p. 225), "sorcier qui suce le sang » est traduit par "imuchuchi intzimumi » ${ }^{48}$. Même si je ne connais pas la signification exacte de ces vocables, il est évident que le terme « sang », " chijabi » ou " nyijia » ${ }^{49}$, n'en fait pas partie. Enfin, en 1559, Gilberti (1901, p. 230), dans son Diccionario en lengua tarasca o de Michoacán, traduit " sorcière qui suce le sang » par " siquame huriata pitsipe », " sorcier qui enlève la chaleur ou souffle vital » ${ }^{50}$. On voit donc que les termes employés pour désigner les sorciers suceurs de sang peuvent être classés en trois groupes :

a) Ceux qui nomment l'action de manger l'âme, le cœur ou le souffle vital des gens.

b) Ceux qui désignent les guérisseurs et les personnages qui «étaient adorés comme des dieux » ou « qui se prenaient pour des dieux ».

c) Ceux qui font référence à l'action de sucer ou consommer des parties ou substances corporelles.

Ce qui montre chez les Espagnols une certaine tendance à réduire une multiplicité d'idées différentes à un seul et même concept. En fait, il est possible que la mention de sorciers suceurs de sang soit le produit d'une interprétation espagnole puisque cette croyance était aussi présente en Europe. Elle est signalée par Las Casas (1967, I, p. 478) dans un récit où il compare les pratiques de sorcellerie de l'Ancien et du Nouveau Monde : «Vicentino dans son Speculo Historial, livre II, chapitre 95, fait aussi référence [à la transformation] et ajoute 
que certains affirment qu'avec des incantations des hommes se changent en oiseaux de nuit comme les hiboux et les chouettes, appelés stryx; ou strigas, en latin, et que la nuit ils sucent le sang des enfants dans les berceaux... " ${ }^{51}$. Les caractéristiques communes au nahualli et à la stryge [vampire tenant de la femme et du chien] - leur supposée capacité de transformation - portent Sahagún et Olmos à croire que les autres traits particuliers des stryx étaient aussi présents chez le nahualli.

\section{Conclusion}

Comme on l'a vu, dans les sources anciennes, les tlahuipuchtin sont définis comme étant des êtres lumineux épouvantables, essentiellement féminins et capables d'enlever leurs jambes. Aujourd'hui, l'activité principale des tlahuipuchtin - ou leur équivalent - est de sucer le sang des enfants. Même si la signification de l'action de s'enlever les jambes reste difficile à comprendre, tout semble indiquer qu'il s'agit d'un symbole de transgression. Le dindon, la forme préférée des tlahuipuchtin actuels, est associé à l'impuissance, la féminité, la pluie, le feu et le tonnerre. Le rituel de transformation du tlahuipuchtli suit d'ailleurs le même schéma que le mythe de la destruction du troisième soleil, c'est le feu qui transforme l'humain en dindon.

Dans la plupart des cas, le tlahuipuchtli contemporain semble incarner ce qui est aberrant et inversé : c'est quelqu'un qui se nourrit du sang des enfants, une femme qui domine un homme, une femme qui sort en cachette pendant la nuit, qui au lieu d'être une bonne chrétienne s'unit avec le diable et a, parfois, des rapports sexuels avec lui. Et c'est à cause de cette image inversée que le tlahuipuchtli a été rapproché de la sorcière espagnole, un être qui représente aussi une morale et un comportement invertis ${ }^{52}$. De sorte que les moyens de neutralisation seraient de nouvelles inversions qui permettent de rétablir le tlahuipuchtli en un être humain normal.

Bien évidemment, il s'agit d'un personnage qui relève de la croyance; en d'autres termes, dans la réalité, personne ne se conçoit comme tlahuipuchtli. Ce n'est pas un spécialiste rituel, mais le fruit d'un imaginaire collectif qui reflète la condamnation sociale d'un comportement vu comme anormal, voire nocif. Parfois, les principaux traits du tlahuipuchtli sont attribués au nahualli, d'autres fois le tlahuipuchtli est considéré comme étant un esprit de la nature - le mazacatl des montagnes de Puebla - et, le plus souvent, il est assimilé à la sorcière espagnole. Le tlahuipuchtli contemporain ne serait donc pas un personnage autonome, mais renverrait à la somme d'une série de traits pouvant être attribués à un autre personnage, le nahualli ou la sorcière. On ne prétend pas que tous les tlahuipuchtin soient des nanahualtin ou inversement, mais que la fonction du tlahuipuchtli est équivalente à celle que l'on attribue aux nanahualtin dans certaines régions. 
Il semble douteux en définitive que la croyance en des nanahualtin suceurs de sang ait existé à l'époque préhispanique. Le fait que le tlahuipuchtli contemporain partage avec le nahualli-tlahuilpuchtli préhispanique son caractère féminin, son association au nahualli, son aspect lumineux, le fait qu'il enlève ses jambes et a une allure épouvantable suggèrent pourtant l'existence d'une fonction chez l'homme-nahualli ancien semblable à celle du tlahuipuchtli moderne. Il est possible qu'il soit censé avoir une autre action, comme celle de manger l'âme des gens peut-être, provoquant également la mort des individus. En tout cas, l'influence de la sorcellerie européenne sur l'image du nahualli-tlahuipuchtli paraît avoir donné lieu à un nouveau personnage, produit du mélange entre le tlahuipuchtli de l'époque préhispanique et la stryge européenne. *

* Manuscrit reçu en septembre 2005, accepté pour publication en février 2006.

\section{NoTES}

Remerciements : Même si j'assume l'entière responsabilité de ce qui est écrit dans ce texte, je dois bien admettre que sa conception n'aurait pas pu être réalisée sans l'inestimable aide de Michel Graulich, de Sybille de Pury, de Danièle Dehouve, d'Erika Araiza, de Francisco Lugo, de Refugio Miranda, de Jacques Galinier et de Roberte Hamayon. Je remercie aussi le CONACYT et le programme de bourses postdoctorales de l'UNAM pour leur soutien économique et logistique.

1. Ce qui peut être confirmé par le fait que le terme tlahuipuchtli servait aussi à désigner des insectes lumineux (Codex de Florence, XI, p. 100).

2. Généralement, dans le contexte mésoaméricain, le terme nahualli (qui se dit aussi nagual ou nahual) est employé pour faire référence à deux notions différentes : il fait référence, d'une part, à un certain type de spécialistes rituels, mentionnés dans les sources comme étant capables de changer de forme à volonté et, d'autre part, à une sorte d'alter ego (ou double animal) qui se trouve tellement lié à l'identité des individus que sa mort entraînerait simultanément la mort de son correspondant humain.

3. Serna (1953, II, pp. 169) traduit mometzcopinani (ou mometzcopinqui) par «celles à qui l'on arracha les jambes "; Key et Ritchie (1953) traduisent le verbe metzcopina par " se désarticuler les os des jambes ". Pourtant, selon López Austin (1967, p. 92) et de Pury (2003, communication personnelle), le terme mometzcopina (ou mometzcopinqui) n'implique ni la désarticulation des os, ni l'arrachement des jambes; le terme en question veut seulement dire « celui qui s'enlève les jambes ".

4. Dans cette variante dialectale, la racine tlahui, de tlahuia " allumer ", est remplacée par tlahuel, de tlahueliloc « méchant, maléfique " (Molina 1970).

5. Ce terme, d'après Wimmer (2003), signifie à la fois " sifflet avec lequel jouent les enfants » et «chouette». Le plus intéressant est que, selon Álvarez Heidenreich (1987, p. 199), "les nahuallisorciers sont facilement reconnaissables parce qu'ils s'approchent des gens en produisant un bruit semblable au bourdonnement d'une abeille, ils produisent ce bruit parce qu'ils arrivent toujours en volant ". De la sorte, le mot chixtle dont la signification est liée au fait de siffler pourrait renvoyer aux caractéristiques attribuées au nahualli dans cette région.

6. Les informations apportées par cet auteur semblent être contradictoires. En effet, même si, dans le travail cité, il ne mentionne jamais le tlahuipuchtli comme un type de nahualli, dix-neuf ans après, Fabregas Puig (1998, p. 188) affirme que « La nahuala est un être craint. Elle reçoit le nom nahua de Tlahuilpoche... ". Nous ne savons pas avec certitude qui, de Fabregas Puig ou des informateurs nahua de la région qu'il étudie, appelle nahuala le tlahuipuchtli.

7. Chez les Nahua de Cuetzalan, la situation est beaucoup plus complexe que dans les autres régions nahua car, d'après Signorini et Lupo (1989, pp. 86-87), personne n'est d'accord sur l'aspect humain des suceurs de sang. Certains de leurs informateurs disent qu'il s'agit d'un type de ehecame (des 
esprits de la nature amoraux et dangereux pour les êtres humains), tandis que d'autres l'identifient avec le nahualli. Segre (1987, p. 84) affirme que "mazaca est aussi la sorcière qui désarticule ses jambes jusqu'aux genoux et vole ». De plus, Signorini et Lupo (ibid.) précisent que les Nahua de Cuetzalan considèrent qu'il existe un autre type de mazacat : il s'agirait d'un être burlesque de nature lumineuse qui a la capacité de disparaître et de changer de forme à volonté, qui n'est jamais confondu avec le nahualli-suceur de sang. D'après Carrasco (1960, p. 111), chez les Chontal de Oaxaca, les suceurs de sang ne sont pas considérés comme des sorciers, mais comme des esprits de la nature qui agissent sous les ordres des sorciers : « Il existe un esprit mauvais qui suce et mord ses victimes [...] ce mauvais esprit tellurique fait ça à cause d'un sorcier ».

8. Ce personnage est présent chez les Otomi (Tranfo 1974, p. 238 ; Galinier 1990, p. 599) sous le nom de nagual ou a'skayo « héron ", chez les Mixe sous le nom de ko :zpi (Lipp 1991, p. 160), chez les Pame sous celui de ganjáo gankjI « bois son sang » (Chemin Bässler 1984, p. 198), parmi les Mazahua (Morales Sales 1985, p. 227), les Zapotèques (Parsons 1966, p. 131), les Totonaques (Ichon 1969, p. 175) et les Popoloca (Jäklein 1974, p. 263) sous celui de bruja « sorcière ».

9. Un enfant de la banlieue de Mexico mentionne que, dans les contes d'épouvante, les sorcières se changent en boules de feu.

10. Par exemple, Francisco Lugo (2003, communication personnelle), doctorant en anthropologie, originaire de Monterrey, raconte que sa grand-mère disait avoir capturé deux sorcières suceuses de sang qui avaient pris la forme de poules.

11. La transformation des tlahuipuchtin n'est pas identique chez tous les Mésoaméricains : chez les Mixe (Lipp 1991, p. 161) et les Mayas du Belize (Thompson 1927-1932, p. 158), « on dit qu’à minuit les sorciers vont à la croisée des chemins, se déshabillent et enlèvent leur tête ». Pour reprendre leur forme humaine, il leur suffit de remettre leur tête. D'après les Otomi d'El Mezquital, pour se transformer, la sorcière « devait tuer un chat et, après minuit et avant que le coq ne chante, elle devait le saigner à un carrefour. Une fois qu'elle avait fait cela, elle demandait au chat la permission de prendre sa forme " (Tranfo 1974, p. 239). Tandis que, dans la croyance zapotèque, pour se transformer, « la sorcière va d'abord à l'église prier Dieu (pedir Dios), à la rivière [et] sur un monticule de cendres dans lesquelles, après s'être déshabillée, elle roule et immédiatement prend sa forme animale. Puis, elle va sucer le sang des gens... » (Parsons 1966, p. 132).

12. Ce sont les autorités qui tuent tlahuipuchtli au sud de Veracruz (Münch 1994, pp. 172-173), elle est assassinée par son conjoint à Chalco-Amecameca (Fabregas Puig 1969, p. 108) et au Morelos (Barrios 1949-1957, p. 59), le mari l'a laissée mourir à Milpa Alta (Madsen 1960, p. 203) et chez les Nahua de la région de Cuetzalan (Segre 1990, p. 405). On retrouve dans le sud Veracruz un récit presque identique, excepté que dans cette région les rôles sont inversés : c'est l'homme qui suce le sang et la femme qui finit par vaincre son mari (Münch 1994, pp. 172-173). Cependant, il existe un autre conte, dans cette même région, où la femme-sorcière (non-tlahuipuchtli) est aussi capturée par son mari (Münch ibid. ; García de Léon 1969, p. 287).

13. Olivier (1997, p. 264) a compilé de nombreuses images qui montrent le caractère unijambiste de ce dieu. Tepeyollotl, dans le Codex Borbonicus (1988, p. 3), Itztli, dans le Codex Borgia (1963, p. 14) et Huitzilopochtli, dans le Teocalli de la guerre sacrée, sont aussi représentés avec un pied en moins. Enfin, dans les Anales de Cuauhtitlan (1945, p. 5), les géants sont appelés tzocuilicxeque ou tzocuilicxime « ceux qui ont des pattes de chardonnerets ». Selon les informateurs de Sahagún (1938, pp. 50-51), le géant était l'un des doubles-nahualli de Tezcatlipoca.

14. Le Codex Telleriano-Remensis (1995, 5r, p. 13) dit explicitement qu'« ils ne représentent pas ici Tezcatlipoca avec le pied cassé, parce qu'ils disent que cette fète [Panquetzaliztli] avait lieu avant qu'il ne devienne pécheur... ".

15. Aucun récit ne dit de façon explicite que Tezcatlipoca est castré ; il est en quelque sorte efféminé, puisqu'on l'appelle cuiloni, c'est-à-dire " sodomite » (Codex de Florence, 1v, p. 35), mais je ne crois pas que cela ait à voir avec la castration.

16. Selon Montoya Briones (1964, p. 174), dans la Sierra Norte de Puebla, la seule espèce mentionnée est le dindon. Chez les Nahua de Tlaxcala, Nutini et Forbes (1987, p. 341) parlent d'une grande 
variété de formes, mais ils spécifient que « l'animal préféré est le dindon et c'est sous cette forme que les tlahuelpuchis doivent sucer le sang des bébés " ; en effet $75 \%$ des 300 légendes provenant de Tlaxcala parlent de dindons (Nutini et Roberts 1993, p. 54). Selon Fabregas Puig (1969, p. 107), les brujas de la région de Chalco-Amecameca se changent en "chat, dindon, poule et zopilote». Une exception notable est celle des Nahua du Guerrero qui, d'après Goloubinoff (1994, p. 579), prennent « l'apparence d'un gros cochon pitzonahuahli, d'un grand chien ou encore d'un animal monstrueux comme, par exemple, un âne sans tête... ". Concernant les Mixe, Lipp (1991, p. 160) précise qu'" à Chiltepec, les sorciers voyagent [sous la forme] de vigoureuses boules de lumière [...], tandis qu'à Ixcatlan ils prennent la forme de cochons, d'ânes, de chats ou de chiens ".

17. Le Codex Telleriano-Remensis (1995, planche 26) dit que le chalchiuhtotolin « est la même chose que le Diable avant le déluge ou Tezcatlipoca qui veut dire miroir fumant... ». Le Códice Vaticano 3738 (1966, lam. XL, p. 96) signale qu'« ils représentaient Tezcatlipoca avec un pied de coq [Molina (1970) traduit huexolotl "dindon" par "coq"] ce qui fait référence à son nom... ". La divinité en question apparaît sous forme de dindon ou habillée en dindon dans le Codex Borbonicus (1988, p. 17), le Codex Telleriano-Remensis (1995, planche 26) et le Codex Borgia (1963, p. 64).

18. D'après Erika Araiza (2004, communication personnelle), ethnologue et sociologue, cette pratique est aussi présente dans la région d'Ecatepec, banlieue nord de Mexico.

19. Galinier (1984, p. 50) signale que, pour les Otomi, le dindon est aussi « associé à la divinité du feu ").

20. Voir Barrios (1949-1957, p. 59), Madsen (1960, pp. 193, 196), Buchler (1980, p. 8) et Nutini et Roberts (1993, pp. 58-59).

21. Actuellement, le dindon est une oftrande typique à «San Juan del Monte ou des esprits de la montagne ; et chez plusieurs peuples du nord de Veracruz il n'y a rien de plus efficace [que le sacrifice d'un dindon] pour demander la pluie » (Noriega Orozco 1997, p. 562).

22. Les Chinantèques et les Popoluca contemporains identifient également le dindon avec le vent qui attire le tonnerre et la pluie (Weitlaner 1981, pp. 97-98 ; Foster 1945-1951, p. 199).

23. Par exemple, Fabregas Puig (1969, p. 107) explique que les brujas de la région de ChalcoAmecameca « portent une marmite pleine de charbon brûlant entre les bras, qu'elles soufllent à travers les petites fenêtres de la marmite. Chaque fois qu'elles soufllent, une lumière très intense se produit... " . Pour la Sierra Norte de Puebla, Montoya Briones (1964, p. 174) affirme que « les tlahuipuchtin peuvent être reconnus par le feu qu'ils portent et par la lumière qu'ils émettent... ». Chamoux (1989, p. 308) précise que les Nahua de la même région considèrent que les tlahuipuchtin sont des êtres de lumière.

24. De plus, Nutini et Roberts $(1993,63)$ et Signorini et Lupo $(1989,86)$ affirment que les tlahuipuchtin de Tlaxcala et les mazacame des montagnes de Puebla peuvent aussi utiliser leur pouvoir hypnotique « pour entrer chez leurs victimes durant la nuit, les faire se lever, marcher jusqu'à un endroit dangereux, comme un précipice, et les faire sauter dans le vide... ».

25. Il est possible que cette méthode ait été utilisée particulièrement contre les tlahuipuchtin ; car ce terme est l'un des plus mentionnés dans cette citation.

26. González Jácome (1997, p. 480) précise que les Nahua contemporains de Xiloxoxtla, Tlaxcala, considèrent que la méthode du miroir ou du miroir d'eau est efficace parce que les tlahuilpuches " s'effraient quand ils regardent le reflet de leur propre image ». Chez les Mixe aussi, un individu peut se protéger des tlahuipuchtin «en plaçant un grand miroir sur une jarre sous le lit... » (Lipp 1991, p. 160).

27. Vu que les Mésoaméricains n'ont commencé à travailler et utiliser couramment les métaux qu'à partir de l'arrivée des Espagnols, il est possible que ce matériel soit conçu par les indigènes contemporains comme un signe de « civilisation ». Ainsi, si cette proposition est juste, la peur des métaux qu'on attribue au tlahuipuchtli et à d'autres types de nanahualtin correspond au caractère sauvage ou non humain du personnage. Dans cette même veine, on rappellera que, d'après Muñoz Camargo (1998, pp. 77, 79), les Chichimèques - qualifiés de « sorciers et nécromanciens » - "buvaient et suçaient le sang des animaux qu'ils tuaient $"$. 
28. Cette croyance s'appuie probablement sur les inversions dont parle le Magnificat : «[...] il renverse les puissants de leur trône, il élève les humbles ; il comble de biens les affamés, renvoie les riches les mains vides ".

29. Il existe néanmoins une certaine tendance à dire qu'ils sont dans les villages voisins. Par exemple, Madsen (1960, p. 203) précise que le seul tlahuipuchtli connu à Tecospa - région de Milpa Alta - est un habitant de Ohtenco marié avec une fille du village; Montoya Briones (1964, p. 174) rapporte que les gens du village de Atla - dans les montagnes de Puebla - estiment que les tlahuipuchtin abondent à Xolotla et San Pablito (un village otomi) ; Nutini et Roberts (1993, p. 55) aflirment eux aussi que "les gens tendent, de façon chauviniste, à situer les tlahuelpuchis plus dans d'autres communautés que dans les leurs. Au niveau régional, les petits villages de Olintla et Hueytochco [...] sont célèbres et universellement connus comme étant des endroits de résidence d'innombrables tlahuelpuchis ».

30. Les Otomi d'El Mezquital, Hidalgo, caractérisent le nagual-tlahuipuchtli comme une femme sans mari. Selon Tranfo (1974, p. 238), « seules les femmes ont la capacité de se transformer en nagual ; les personnes que l'on soupçonne [de se transformer en nagual] sont des vieilles femmes, presque toujours célibataires ou veuves [...]. Ces femmes se transforment en animal, c'est-à-dire qu'elles prennent la forme du nagual qui leur est particulier, uniquement pendant la nuit, et sortent vagabonder en commettant des crimes parmi lesquels le pire est celui de sucer le sang des enfants $"$. Chez les Mixtèques, une femme accusée d'être nagual-suceur de sang avait une apparence peu commune - car elle était grande et obèse - et avait une occupation peu habituelle pour les femmes : elle vendait de l'eau-de-vie (Kaplan 1956, p. 366). Pour les Mazahua, "les sorcières sont des femmes postménopausiques devenues dangereuses parce qu'elles ont perdu leur capacité reproductrice, mais elles n'ont pas perdu leur besoin de sperme » (Oemichen 2002, p. 139). Chez les Purépecha, les sorcières sakuapiri, « femmes de la rue », sont toujours imaginées comme des femmes sans mari. " Généralement celles-ci sont, soit des vieilles femmes sans famille, soit des femmes, souvent veuves, considérées comme des prostituées » (Beals 1946, p. 156). Comme on le voit dans le récit initial, la plupart du temps, les tlahuipuchtin sont des femmes: selon Fabregas Puig (1969, p. 107), dans la région de ChalcoAmecameca, les tlahuipuchtin sont exclusivement des femmes; Nutini et Forbes (1987, p. 341) affirment qu'à Tlaxcala « la plupart des tlahuelpuchis sont des femmes et on considère que celles-ci sont plus maléfiques, plus puissantes et plus sanguinaires que les hommes ». Même si, a priori, les tlahuipuchtin peuvent être des hommes ou des femmes, tous les récits provenant de la vallée de Puebla parlent de femmes-suceuses de sang (Fagetti 1996, p. 219). Chamoux (1989, p. 308) dit que, pour les Nahua de Huauchinango, les tlahuipuchtin peuvent être aussi bien des hommes que des femmes, tandis que, selon Münch (1994, pp. 172-173), au sud de Veracruz, les tlahuipuchtin - appelés brujos - sont uniquement des hommes. Pour les Mazahua (Morales Sales 1985, p. 229), les Otomi d'El Mezquital (Tranfo 1974, p. 239) et les Zapotèques (Parsons 1966, p. 131), les tlahuipuchtin sont exclusivement des femmes. Galinier (1990, p. 647) affirme que, pour certains peuples otomi, les tlahuipuchtin sont presque toujours des femmes.

31. Nutini et Roberts $(1993$, pp. 55,74$)$ affirment que, sous leur forme humaine, les tlahuipuchtin sont des femmes normales, «à part le fait qu'elles sont considérées comme étant colériques (geniudas [mot espagnol du Mexique qui qualifie les femmes qui se fàchent facilement]), très dominantes et possessives... ». On suppose qu'il y a chez les tlahuipuchtin « une complète inversion dans la relation homme-femme ».

32. Le seul homicide connu d'un tlahuipuchtli est rapporté par Nutini (Nutini et Roberts 1993, p. 74) qui affirme avoir été témoin de ce fait. Cependant, il faut signaler qu'il s'agissait d'une situation hors du commun, un moment de crise, car il y avait eu plusieurs enfants qui étaient morts durant les nuits précédentes sans que l'on puisse établir la cause de leur décès.

33. Les réunions secrètes de tlahuipuchtin sont aussi mentionnées par Montoya Briones (1964, p. 174), Gamio (1922, p. 307) et Mendoza (1952, p. 288). Il faut remarquer que, même si la croyance aux sabbats des tlahuipuchtin est très répandue, elle n'est pas universelle. La seule région connue où cet élément est absent est la vallée de Tlaxcala. Nutini et Roberts (1993, pp. 36, 61) affirment qu'« il n'y a 
aucun type de réunion [de tlahuelpuchis] [...], les gens regardent les tlahuelpuchis comme étant des agents indépendants, non reliés à aucun autre être surnaturel maléfique plus puissant... ". Cependant, dans cette région, l'image de la sorcière a été remplacée par celle du vampire européen ; cette influence est évidente dans le fait que l'on considère qu' il n'y a que deux choses qui peuvent offrir une vraie protection et empêcher que les enfants soient sucés par les tlahuelpuchis : l'ail et l'oignon » (ibid., p. 70).

34. Gamio (1922, p. 307) présente un récit, presque identique, où une sorcière et son mari vont rendre visite au démon à l'intérieur d'une caverne. La sorcière du récit conseillait à son mari - qui assistait pour la première fois à la réunion : « On va bientôt arriver là où l'on va. De cette caverne sortira un homme que l'on adore ; ne t'effraye pas, ne mentionne pas Dieu [...]. Et lui [le mari], en regardant [un homme] très épouvantable, s'effraya et dit : “Ave Maria purisima ! Jésus !" [...] les ennemis s'enfuirent vers l'intérieur de la caverne... ». De leur côté, Signorini et Lupo (1989, p. 87) présentent un autre épisode où les mazacame s'enfuirent uniquement parce qu'un individu dit « au nom de Dieu ! ».

35. D'après Dehouve (2004, p. 232), les prières et les mentions de Dieu et des saints qui font disparaître le démon et les animaux diaboliques apparaissent en Europe dans des exempla du XII ${ }^{\circ}$ siècle. Elle signale que « d'un point de vue indien, il est possible d'identifier des nahuales dans les animaux diaboliques $"$.

36. En comparant le tlahuipuchtli nahuatl contemporain avec la bruja « sorcière " de Monterrey - dans un contexte complètement urbain où l'on ne parle aucune langue indigène et, a priori, plus proche de la croyance espagnole -, on trouve les différences suivantes: la bruja ne s'enlève pas les jambes pour effectuer sa métamorphose, elle remplace ses yeux par ceux d'un chat, elle ne se change pas en dindon, mais en chouette, et ce n'est pas son destin qui détermine sa condition de bruja mais un pacte démoniaque (Francisco Lugo 2004, communication personnelle).

37. En 1594, une mulâtresse du Veracruz fut accusée d'être sorcière et de voler dans les airs pendant la nuit (AGN Inquisición 1594, 206.9, fol. 290rv). Il est dit, en 1737, d'une autre mulâtresse, appelée María Nieves Montiel, qu'elle était sorcière et qu'elle volait en une seule nuit de Guatemala à Lima (AGN Inquisición 1737, 864. s/n, fol. 312r).

38. "Sorcière » est le mot qu'utilise l'auteur et/ou ses informateurs pour traduire le terme nahuatl " tlahuipuchtli ».

39. D'après Miranda, « on dit que si nous tuons un nahualli [jeteur de sorts], nous devons le brûler [...]. Parce que si on ne le brûle pas, son ombre, son esprit ou son âme, tel que nous pouvons l'appeler, peut s'introduire dans - je ne sais pas - l'un de nos chiens, même peut-être dans l'un des membres de notre famille et eux, ils nous feraient du mal "). C'est vrai que, d'après les mythes, la déesse Itzpapalotl fut brûlée par Iztac Mixcoatl ; pourtant, les Anales de Cuauhtitlan (1945, p. 3) spécifient qu'il lui envoya auparavant des flèches.

40. En effet, au Xvir ${ }^{\mathrm{e}}$ siècle, Castañega $(1994$, p. 19) affirmait à propos de la sorcière espagnole que " ce nom bruxa [sorcière] est un vocable italien qui veut dire brûlé ou brûlée, parce que la peine infligée à de tels magiciens et enchanteurs était celle de les brûler, c'est pour cela que ce nom leur a été attribué... ". Le personnage de la sorcière est donc, en soi, étroitement associé au type de punition.

41. Les seules exceptions que j'ai pu trouver sont le tlahuipuchtli de Chalco-Amecameca et le nahual de Xalitla, Guerrero. D’après Fabregas Puig (1969, p. 111), la tlahuipuchtli de la région de ChalcoAmecameca obtient ses pouvoirs, de la même façon que la sorcière espagnole, par un pacte avec le démon, pacte qui " est consolidé au moyen d'une relation sexuelle ». Tandis que Goloubinoff (1994, p. 579) affirme qu'à Xalitla, Guerrero, « il s'agit d'un homme ou d'une femme ayant acquis le pouvoir de se métamorphoser grâce à des pratiques magiques apprises par d'autres ou dans des livres ». Le cas des mazakame des montagnes de Puebla est assez contradictoire car, malgré le caractère inné de leur activité, dans l'un des récits de Segre (1990, pp. 404-405), il est fait mention d'un prêtre catholique qui explique au mari d'une mazacatl : « Ta femme n'est pas une personne, c'est le diable. C'est une personne parce qu'elle a été créée par Dieu, elle est humaine, mais elle veut être un diable, c'est pour cela qu'elle devient un diable, elle fait ce qu'elle veut [...]. Laisse-la, elle va mourir... ".

42. Dans l'espagnol actuellement parlé au Mexique et, probablement, en Espagne, la phrase " chupa a los niños" est très souvent interprétée comme « suce le sang des enfants». On observe par 
ailleurs que, dans le langage populaire de la ville de Mexico, un synonyme de "mourir » est "ser chupado por la bruja ", "être sucé par la sorcière ".

43. Olmos (1990, pp. 68-69), dit que les nahuales sont « des vieilles femmes qui font naître des petits enfants chéris, pour leur sucer le sang [...]. Le diable enseigne beaucoup de choses inquiétantes aux sages-femmes, à celles qui font naître les enfants, de sorte qu'elles ne consomment que leur sang [celui des enfants] ». Néanmoins, on sait que le texte d'Olmos n'est qu'une adaptation en nahuatl d'une œuvre espagnole sur la sorcellerie. Le démon « fait que les prêtres, de la manière la plus subtile et secrète qu'ils peuvent, tuent des enfants comme le font plusieurs sages-femmes sorcières ou sucent du sang humain par des moyens secrets et cauteleux que le démon leur enseigne » (Castañega 1994, fol.18r, p. 27).

44. Comme en témoignent les informateurs de Sahagún (1946-1947, pp. 151, 241-242; Codex de Florence 1959, I, p. 4, x, p. 29 ; López Austin 1967, p. 110), ce terme ne désignait pas une quelconque action maléfique, mais l'action de sucer les corps malades pour en extraire des objets pathogènes.

45. Composé de nogue " manger » et nate " esprit ou souffle » (Urbano 1990, p. 202 ; Diccionario castellano-otomi... 1956, pp. 222, 261).

46. Mamuayte, " cœur ou âme " (Urbano 1990, pp. 98, 28).

47. Nêyethi « chose médicale », anteyethhi « médicament », ñethi « médecine, remède » (Urbano 1990, p. 206 ; Diccionario castellano-otomi... 1956, p. 236).

48. Chuchi « mâcher, sucer », tzitzi « manger » (Escalante Hernández 1997, pp. 31, 129).

49. Deux termes dérivés de la racine $j$ 'abi qui signifie « jour, âme, esprit, temps» (Escalante Hernández 1997, pp. 24, 38 ; Reynoso González 1998, p. 82).

50. Siquame signifie « devin, sorcier, ensorceleur, magicien, chamane » (Velázquez Gallardo 1978, p. 184). Huriata, « soleil », semble partager la même racine que huriaqua « jour » et hiretequa « esprit ou souffle »; les termes dérivés de la racine pitz ont une signification proche de "enlever, quitter, lâcher " (Gilberti 1901, pp. 28, 45, 86).

51. Voir aussi Caro Baroja (1972, pp. 55-56) et Rodríguez-Virgil (1996, p. 84).

52. Rappelons que, pour la scholastique, le sabbat était une véritable inversion du rituel chrétien. À ce propos, Castañega $(1994$, p. 16) aftirme que la cérémonie diabolique « contrefaisait les sacrements, comme si elle se moquait d'eux... ", tandis que, dans le procès inquisitorial des sorciers de Zugarramurdi (in Proceso a la brujería... 1989, p. 56), on dit parfois que les sorciers « se confessent devant lui [le démon] et s'accusent de certains péchés, comme celui d'être entré dans une église, d'[avoir] écouté des messes et d'autres choses que font les chrétiens, mais aussi des maux qu'ils n'ont pas commis quand ils pouvaient les faire ».

\begin{tabular}{ll} 
& \multicolumn{1}{c}{ Abréviations } \\
AHDCH & $\begin{array}{l}\text { Archivo Histórico Diocesano de Chiapas } \\
\text { Archivo General de la Nación } \\
\text { AGN }\end{array}$ \\
CEMCA & Centre d'études mexicaines et centre-américaines \\
CNRS & Consejo Nacional para la Cultura y las Artes \\
CONACULTA & Estudios de Cultura Náhuatl \\
ECN & École des Hautes études en sciences sociales \\
EHESS & Escuela Nacional de Antropología e Historia \\
ENAH & Fondo de Cultura Económica \\
FCE & Instituto de Investigaciones Antropológicas \\
IIA & Instituto de Investigaciones Filológicas \\
IIF & Instituto de Investigaciones Históricas \\
IIH & Instituto Nacional Indigenista \\
INI & Instituto Nacional de Antropología e Historia \\
INAH &
\end{tabular}




$\begin{array}{ll}J S A & \text { Journal de la Société des Américanistes } \\ \text { JAF } & \text { Journal of American Folklore } \\ \text { SAF } & \text { Secretaría de Agricultura y Fomento } \\ \text { SEP } & \text { Secretaría de Educación Pública } \\ \text { SHCP } & \text { Secretaría de Hacienda y Crédito Público } \\ \text { SIW } & \text { Smithsonian Institution of Washington } \\ \text { SMA } & \text { Sociedad Mexicana de Antropología } \\ \text { UAEM } & \text { Universidad Autónoma del Estado de México } \\ \text { UAM } & \text { Universidad Autónoma Metropolitana } \\ \text { UAP } & \text { University of Arizona Press } \\ \text { UCP } & \text { University of California Press } \\ \text { UNAM } & \text { Universidad Nacional Autónoma de México } \\ \text { UTP } & \text { University of Texas Press }\end{array}$

\section{SOURCES CONSULTÉES}

Documents de l'Archivo General de la Nación

AGN. Inquisición. 206.9. Proceso contra Andrés de Villalobos, 1594, Veracruz.

AGN. Inquisición. 864.s/n. Proceso contra María de Nieves Montiel, 1737, Granada, Guatemala.

AGN. Inquisición. 939.8. Declaración de María Borrego, 1751, Santiago de Monclova.

Documents de l'Archivo Histórico Diocesano de Chiapas

Sobre idólatras del pueblo de San Andrés, Partido de Chamula, 1778, San Andrés Larráinzar.

\section{RÉFÉRENCES CITÉES}

Álvarez Heydenreich Laurencia

1987 La enfermedad y la cosmovisión en Hueyapan, Morelos, INI, Mexico.

Anales de Cuauhtitlan

$1945 \quad$ Voir Codex Chimalpopoca

BAutista Juan Fray

1965 "Algunas abusiones antiguas », in Angel María Garibay (éd.), Teogonía e historia de los mexicanos. Tres opúsculos del siglo XVI, Ed. Porrúa, Mexico, pp. 140-156.

Barrios E. Miguel

1949-1957 «Textos de Hueyapan, Morelos », Tlalocan, III, pp. 53-75, Mexico.

BEALS Ralph

1946

Cheran. A Sierra Tarascan village, SIW, Social Anthropology, Washington. 


\section{BUCHLER Ira}

1980 "Nagualism : a structural sketch of tales from a Mexican village », Anthropology, IV (2), pp. 1-14, State University of New York, New York.

Campos Julieta

1982 La herencia obstinada. Análisis de cuentos nahuas, FCE, Mexico.

\section{Caro Baroja Julio}

1972 Les sorcières et leur monde, traduit par M.- A. Sarrailh, Gallimard, Paris.

\section{Carrasco Pedro}

$1950 \quad$ Los otomis. Cultura e historia prehispánicas de los pueblos mesoamericanos de habla otomiana, IIH/UNAM-INAH, Mexico.

1960 Pagan rituals and beliefs among the Chontal indians of Oaxaca, Mexico, UCP, Anthropological records, Berkeley/Los Angeles.

\section{CASTAÑega Martín de}

1994 Tratado muy sútil y bien fundado de las supersticiones y hechicerías y varios conjuros $y$ abusiones $y$ otras cosas al caso tocantes y de la posibilidad y remedio de ellas, édité et introduit par Juan Robert Maro Abad, Instituto de Estudios Riojanos, Logroño.

\section{Chamoux Marie-Noëlle}

1989 "La notion nahua d'individu : un aspect du tonalli dans la région de Huauchinango, Puebla », in Dominique Michelet (éd.), Enquêtes sur l'Amérique moyenne, INAH/CONACULTA/CEMCA, Mexico, pp. 303310.

\section{CHEMIN BÄssLer Heidi}

1984 Los pames septentrionales de San Luis Potosí, INI, Mexico.

\section{Codex de Florence}

1950-1963 Voir Sahagún.

Codex Telleriano-Remensis : ritual, divination and history in a pictorial Aztec manuscript 1995 édité par Eloise Quiñones Keber, UTP, Austin.

\section{Codex Borbonicus}

1988 édité par Francisco del Paso y Troncoso, Siglo XXI, Mexico.

\section{Codex Borgia}

1963 commentaires d'Eduard Seler, FCE, Mexico.

Codex Chimalpopoca. Anales de Cuauhtitlan y Leyenda de los soles 1945 traduit par Primo Feliciano Velázquez (avec facsimilé), UNAM, Mexico.

Códice Vaticano 3738, Códice Ríos ou Códice Vaticano A Soles

1964-1966 in Antigüedades de México, manuscrits mexicains compilés par Lord Kingsborough, édité par José Corona Núñez, 4 vols, SHCP, Mexico, vol. III, pp. 7-314. 
CORONA NúÑEZ José

1946 «Esquema de deidades de los Tarascos », in Jorge A. Vivo (éd.), 4a Mesa Redonda de la SMA : el Occidente de México, Museo Nacional de Historia, Mexico, p. 139.

Dehouve Danièle

2004 L'évangélisation des Aztèques ou le pécheur universel, Maisonneuve-Larose, Paris.

Diccionario castellano-otomi, otomi-castellano

1956 Instituto Lingüístico de Verano, Cuadernos del Valle del Mezquital/ Instituto Lingüístico de Verano, Itzmiquilpan, Hidalgo.

Escalante Hernández Roberto

1997 Diccionario matlatzinca-español, Colegio de lenguas y literatura indígenas/ Instituto Mexiquense de Cultura, Toluca.

Fabregas Puig Andrés

1969 El nahualismo y su expresión en la región de Chalco-Amecameca, mémoire de maestría en anthropologie, ENAH/INAH/SEP, Mexico.

1998 El mundo de los nahuales : un rasgo de religiosidad popular en el centro de México ", in Angel Espina Barrio (éd.), Antropología en Castilla y León e Iberoamérica. Aspectos generales y religiosidad popular, Instituto de Investigaciones Antropológicas de Castilla y León, Castilla-León, pp. 185195.

FAGETTI Antonella

1996 Cuerpo humano y naturaleza en la cosmovisión de un pueblo campesino, mémoire de maestría en anthropologie, ENAH/INAH/SEP, Mexico.

Foster George

1945-1951 "Sierra Popoluca folklore and beliefs », University of California Publications in American Archaeology and Ethnology, XLII, pp. 177-250, Berkeley/Los Angeles.

GaLinier Jacques

1984 «L’homme sans pied. Métaphores de la castration et imaginaire en Mésoamérique ", L'Homme, XXIV (2), pp. 41-58.

1990 La Mitad del mundo. Cuerpo y cosmos en los rituales otomies, UNAM/CEMCA/INI, Mexico.

GAmio Manual

1922

La población del valle de Teotihuacan. Representativa de los que habitan en zonas rurales del Distrito Federal y de los Estados de Hidalgo, Puebla, México y Tlaxcala, 3 tomes, SAF/Dirección de Antropología, Mexico.

García de León Antonio

1969 «El universo de lo sobrenatural entre los nahuas de Pajapan, Veracruz », ECN, VIII, pp. 279-312, Mexico. 


\section{GiLberti Maturino}

1901 Diccionario en lengua tarasca o de Michoacán, édité par Antonio Peñafiel, Palacio Nacional, Mexico.

\section{Goloubinoff Marina}

1994 Relations sociales et commerce chez les Indiens nahuas du Balsas, thèse de doctorat de $3^{\mathrm{e}}$ cycle, université de Paris X, Nanterre.

GonZÁlez Jícome Alba

1997 "Agricultura y especialistas en la ideología agrícola : Tlaxcala, México », in Beatriz Albores et Johanna Broda (éd.), Graniceros, cosmovisión y meteorología indígenas de Mesoamérica, IIH/UNAM, Mexico, pp. 467-501.

IcHON Alain

1969 La religion des Totonaques de la Sierra, CNRS, Paris.

IxtLiLxóchitl Don Fernad de Alva

1975-1977 Obras históricas, 2 tomes, édités par Edmundo O'Gorman, IIH/UNAM, Mexico.

JÄKLEIN Klaus

1974 Un pueblo popoloca, INI/SEP, Mexico.

JoNGHE Édouard de

1905 "Histoyre du Mechique. Manuscrit français inédit du Xvi siècle d'André Thevet », Journal de la Société des Américanistes de Paris, nouvelle série, II (1), pp. 1-41.

Kaplan N. Lucille

1956 "Tonal and nagual in the coastal Oaxaca, México », JAF, LXIX (274), pp. 363-368, Philadelphie.

Key Harold et Mary Ritchis

1953 Vocabulario mejicano de la Sierra de Zacapoaxtla, Puebla, Instituto Lingüístico de Verano/Dirección General de Asuntos Indígenas, Mexico.

Las CASAs Bartolomé de

1967 Apologética historia sumaria, édition préparée par Edmundo O’Gorman, avec une étude préliminaire, des appendices et un index thématique, IIH/UNAM, Mexico.

Leyenda de los soles

1945 Voir Codex Chimalpopoca. Anales de Cuauhtitlan.

Lipp Frank J.

1991 The Mixe of Oaxaca. Religion, ritual and healing, UTP, Austin.

Lison-Tolosana Carmelo

1996 Las brujas en la historia de España, Ed. Temas de hoy, Collection Bolsitemas, Madrid.

LóPEz Austin Alfredo

1967 «Cuarenta clases de magos en el mundo náhuatl », ECN, VII, pp. 87-117, Mexico. 
Lupo Alessandro

2001 "La cosmovisión de los nahuas de la Sierra de Puebla », in Johanna Broda et Jorge Félix Baez (éd.), Cosmovisión, ritual e identidad de los pueblos indigenas de México, Biblioteca Mexicana/FCE, Mexico, pp. 335-389.

MADSEN William

1960 The Virgin's Children. Life in an Aztec village today, Greenwood Press Publishers, Westport, Connecticut.

MARtínez del Río Amalia

1962 «Leyendas de Tetelcingo », Tlalocan, IV, pp. 80-85, Mexico.

Mendieta Jerónimo de

1945 Historia eclesiástica indiana, Editorial Salvador Chávez Hayhoe, Mexico.

Mendoza R. R. Virginia

1952 "La bruja en México ", in Sol Tax (éd.), Aculturation in the Americas. XXIXth International Congres of Americanists, University of Chicago Press, Chicago, pp. 285-289.

Molina Alonso de

1970 Vocabulario en lengua castellana y mexicana y mexicana y castellana, étude préliminaire de Miguel León-Portilla, fac-similé, Ed. Porrúa, Mexico [1571].

Montoya Briones José de Jesús

1964 Alta, etnografia de un pueblo náhuatl, INAH, departamento de Investigaciones Antropológicas, Mexico.

Morales Sales Edgar Samuel

1985 La tradición oral y la lengua mazahua en el Estado de México, UAEM, Cuadernos de Investigación, Toluca.

MüNCH Guido

1983 "Cosmovisión y medicina tradicional entre los popolucas y nahuas del sur de Veracruz », in Lorenzo Ochoa y Thomas A. Lee Jr (éd.), Antropología e historia de los mixe-zoques y mayas. Homenaje a Franz Blom, UNAM/Brigham Young University, Mexico, pp. 367-381.

1994 Etnología del Istmo veracruzano, IIA/UNAM, Mexico.

Muñoz Camargo Diego

1998 Historia de Tlaxcala : Ms 210 de la Biblioteca Nacional de París, Luis Reyes (paléographie), Gobierno del Estado de Tlaxcala/Universidad Autónoma de Tlaxcala, Centro de investigaciones y estudios superiores en antropología social, Mexico.

Noriega Orozco Blanca Rebeca

1997 «Tlamatines : los controladores de tiempo de la falda de Cofre de Perote, Estado de Veracruz ", in Beatriz Albores et Johanna Broda (éd.), Graniceros, cosmovisión y meteorología indigenas de Mesoamérica, IIH/UNAM, Mexico, pp. 525-563. 


\section{Nutini Hugo et Jean Forbes}

1987 «Nahualismo, control de los elementos y hechicería en Tlaxcala rural », in Susana Glantz (éd.), La heterodoxia recuperada. En torno a Angel Palerm, FCE, Mexico, pp. 321-346.

Nutini Hugo et John RoBerts

1993 Blood-sucking witchcraft. An epistemological study in anthropomorphic supernaturalism in rural Tlaxcala, UAP, Arizona.

\section{Oemichen Cristina}

2002 "Comunidad y cosmovisión entre los mazahuas radicados en la ciudad de México ", Estudios de Cultura Otopame, 3, pp. 123-148, IIA/UNAM, Mexico.

OLIVIER Guilhem

1997 Moqueries et métamorphoses d'un dieu aztèque. Tezcatlipoca, le seigneur au miroir fumant, Institut d'Ethnologie, Paris.

Olmos Andrés de

1990 Tratado de hechicerías y sortilegios, paléographie du texte en nahuatl, version en espagnol, introduction et notes d'Henrico Martínez, avec une étude introductive de Asunción H. de León-Portilla, IIH/UNAM, Mexico.

Parsons Elsie Clews

1966 Mitla. Town of the souls. And other Zapoteco-speaking pueblos of Oaxaca, Mexico, University of Chicago Press, Chicago/Londres.

Proceso a la brujería. En torno al auto de fé de los brujos de Zugarramundi, Logroño 1610 1989 Manuel Fernández Nieto (éd.), Ed. Tecnos, Madrid.

Procesos de indios idólatras y hechiceros

1912 Estados Unidos Mexicanos/Secretaría de Relaciones Exteriores/Publicaciones del AGN, Mexico.

Pury Sybille de

1982 "Cuentos y cantos de Tlaxcalancingo, Puebla », Tlalocan, IX, pp. 71-105, Mexico.

Relación de Michoacán.

1977 José Corona Núñez (éd.), Ed. Balsal, Morelia.

Reynoso GonZÁlez Elpidia

1998 Vocabulario español-tlahuica, Instituto Mexiquense de Cultura/Colegio de lenguas y literatura indígenas, Toluca.

RoBe Stanley L.

1971 Mexican tales and legends from Veracruz, introduction, classification et notes de Stanley L. Robe, University of California, Los Angeles/ Berkeley/Londres.

Rodríguez-VIRGIL Rubio Juan L.

1996 Bruxas, lobos e inquisición. El proceso de Ana María de Lobera, Ed. Nobel, Oviedo. 
SANDStrom Alan R.

1991 Corn is our blood. Culture and ethnic identity in a contemporary Aztec village, University of Oklahoma Press, Norman/Londres.

\section{SAHAGÚn Bernardino de}

1938 Historia general de las cosas de la Nueva España, Ed. Pedro Robledo, Mexico [1969].

1946-1947 «Paralipómenos de Sahagún», fac-similé de Del Paso y Troncoso, tome VI, cahier 2 (127), traduit par Ángel María Garibay, Tlallocan. A journal of source materials on the native cultures of Mexico, II (2-3), pp. 164174, 135-254, La Casa de Tlaloc, Mexico.

1950-1963 Florentine Codex. General history of the things of New Spain, traduit par Arthur J. O. Anderson et Charles E. Dibble, Monographs of the School of American Research, Santa Fe, New Mexico.

1969 Augurios y abusiones, fuentes indigenas de la cultura náhuatl, textes des informateurs de Sahagún, introduction, version, notes et commentaires d'Alfredo López Austin, IIH/UNAM, Mexico.

SEGRe Enzo

1987 Las máscaras de lo sagrado, INAH, Colección divulgación, Mexico.

1990 Metamórfosis de lo sagrado y de lo profano. Narrativa náhuatl de la Sierra Norte de Puebla, INAH, Colección divulgación, Mexico.

SERna Jacinto de la

1953 "Manual de ministros de indios para el conocimiento de sus idolatrías y extirpación de ellas ", in Tratado de las idolatrías, supersticiones, dioses, ritos, hechicerías y otras costumbres gentílicas de las razas aborigenes de México, vol. I, notes, commentaires et étude préliminaire de Francisco del Paso y Troncoso, Ed. Fuente Cultural, Mexico, pp. 47-368.

Signorini Italo et Alessandro LuPo

1989 Los tres ejes de la vida. Almas, cuerpo, enfermedad entre los nahuas de la Sierra de Puebla, Universidad Veracruzana, Xalapa.

TAGGART James

$1984 \quad$ Nahuat myth and social structure, UTP, Austin.

TEDLock Barbara

1982 Time and the Highland Maya, University of New Mexico Press, Albuquerque.

Tezozómoc Fernand Alvarado

1991 Crónica mexicayotl, traduit du nahuatl par Adrián León, IIH/UNAM, Mexico.

Thompson J. Eric S.

1927-1932 Ethnology of the Mayas of southern and central British Honduras, Field Museum of Natural History, Anthropological Series, XVII (1-4), Chicago.

Torquemada Juan

1943-1944 Monarquía indiana, 3 vols, Editorial Salvador Chávez Hayhoe, Mexico. 
Tranfo Luigi

1974 Vida y magia en un pueblo otomi del Mezquital, INI/SEP, Mexico.

Urbano Alonso

1990 Arte breve de la lengua otomi y vocabulario trilingüe español-nahuatl-otomi, édité par René Acuña, IIF/UNAM, Mexico.

Velázquez Gallardo Pablo

1978 Diccionario de lengua phorhepecha, FCE, Mexico.

Vélez Cervantes Jorge César

1996 «El nahualismo y los tepahtiani/nahualmej de Cuetzalan, Sierra de Puebla ", Alteridades, 6 (12), pp. 33-38, Mexico.

WEITLANER J. Roberto

1981 Relatos mitos y leyendas de la Chinantla, INI, Antropología Social 53, Mexico.

Williams García Roberto

1972 Mitos tepehuas, SepSetentas, SEP, Mexico.

Wimmer Alexis

2003 Dictionnaire nahuatl français [Remi Siméon-Molina], avec la collaboration de Marc Thouvenot, Ed. SUP/INFOR, CNRS/CELIA, Paris [www.supinfor.com].

ZaNTwisk Van Ram

1960 Los indigenas de Milpa Alta, Instituto real de los Trópicos, sección de antropología cultural y física, Amsterdam.

Zavala Mercedes

2001 "Leyendas de la tradición oral del noreste de México », Revista de literaturas populares, I (1), pp. 25-45, Mexico. 